\title{
POSICIONAMENTO CIRÚRGICO: CUIDADOS DE ENFERMAGEM NO TRANSOPERATÓRIO
}

\author{
Surgical positioning: nursing care in the transoperative period \\ Posicionamiento quirúrgico: cuidados de la enfermería en el período transoperatorio \\ Amanda Braz Miranda', Amanda Rosa Fogaça², Mariane Rizzetto³, Laura Cristina Cuvello Lopes ${ }^{4}$
}

RESUMO: Durante a atuação em centro cirúrgico observou-se que a enfermagem não atuava no posicionamento do paciente na mesa cirúrgica, o que nos incentivou a investigar sobre o tema. Objetivo: Identificar os cuidados de enfermagem no posicionamento, relatando as complicações. Método: Trata-se de revisão integrativa da literatura, com busca nos bancos de dados LILACS (Literatura Latino-Americana e do Caribe em Ciências da Saúde) e SciELO (Scientific Electronic Library Online), no período de agosto de 2004 a setembro de 2014. Resultados: Foram encontrados 20 artigos e selecionados 10 conforme critérios. Conclusão: O posicionamento pode ocasionar complicações, sendo a úlcera por pressão a mais frequentemente apontada. As intervenções eficazes são aquelas que aliviam as pressões durante a permanência na mesa. O enfermeiro é o responsável pelos cuidados de enfermagem e juntamente com a equipe deve promover ações que garantam a segurança do paciente, considerando as particularidades e os recursos disponíveis. Palavras-chave: Posicionamento do paciente. Cuidados de enfermagem. Úlcera por pressão.

ABSTRACT: During the operation room activities, it was observed that the nursing team did not actively perform patient positioning on the operating table, which encouraged us to investigate the issue. Objective: To identify the nursing care routine in patient positioning, reporting complications. Methods: This is an integrative literature review, with search in LILACS (Literatura Latino-Americana e do Caribe em Ciências da Saúde) and SciELO (Scientific Electronic Library Online) databases, from August to September 2014. Results: We found 20 articles and selected 10 according to the criteria. Conclusion: The positioning can cause complications, with pressure ulcers being the most frequent. Effective interventions are those that relief the pressure during the patient's stay at the table. The nurse is responsible for nursing care and, together with the team, for actively promoting actions that ensure patient safety, considering the circumstances and available resources.

Keywords: Patient positioning. Nursing care. Pressure ulcer.

RESUMEN: Durante la actuación en el centro quirúrgico, se observó que la enfermería no trabajaba en el posicionamiento del paciente en la mesa quirúrgica, lo que alentó a investigar la cuestión. Objetivo: Identificar los cuidados de enfermería en el posicionamiento, haciendo un informe sobre las complicaciones. Método: Se trata de una revisión integradora de la literatura, utilizándose la búsqueda en las bases de datos LILACS (Literatura LatinoAmericana e do Caribe em Ciências da Saúde) y SciELO (Scientific Electronic Library Online), en el período comprendido entre agosto de 2004 hasta septiembre de 2014. Resultados: Se encontraron 20 artículos y se seleccionaron 10 de ellos, basándose en criterios. Conclusión: El posicionamiento puede causar complicaciones, y las ulceras por presión son las más frecuentes reportadas. Las intervenciones eficaces se relacionan con el alivio de las presiones en la mesa quirúrgica. El enfermero es responsable por los cuidados de enfermería y debe promover acciones juntamente con su equipo que garanticen la seguridad del paciente, teniendo en cuenta las particularidades y los recursos disponibles.

Palabras clave: Posicionamiento del paciente. Cuidados de enfermería. Úlcera por presión.

'Graduada pelo Centro Universitário Ítalo Brasileiro; enfermeira trainee no Amato Instituto de Medicina Avançada - São Paulo (SP), Brasil. E-mail: amdrdg@hotmail.com Rua Padre Joaquim Correia de Almeida, 200 - Parque Arariba - CEP: 05778-280 - São Paulo (SP), Brasil.

${ }^{2}$ Graduada pelo Centro Universitário Ítalo Brasileiro; enfermeira júnior no Hospital Municipal do M'Boi Mirim - São Paulo (SP), Brasil. E-mail: amanda.vtr@hotmail.com

${ }^{3}$ Especialista em Psiquiatria e Dependência Química pela Universidade de São Paulo; docente do Centro Universitário Ítalo Brasileiro - São Paulo (SP), Brasil. E-mail: mariane.rizzetto@uniitalo.it

4Doutora em Ciências pela UNIFESP; docente do curso de Enfermagem do Centro Universitário Ítalo Brasileiro - São Paulo (SP), Brasil. E-mail: laura.cuvello@uniitalo.edu.br

Recebido: 24 jan. 2016 - Aceito: 21 mar. 2016

DOI: $10.5327 / 21414-4425201600010008$ 


\section{INTRODUÇÃO}

O termo enfermagem perioperatória é um conceito que vem se firmando no Brasil. Seu sentido é amplo, abrangendo os períodos pré-operatório, transoperatório e pós-operatório ${ }^{1}$. Nesse contexto a enfermagem perioperatória fundamenta-se em seis princípios: integralidade, individualidade, participação, continuidade, documentação e avaliação².

O enfermeiro é o responsável pelo planejamento e pela implementação de intervenções de enfermagem que previnam as complicações decorrentes do procedimento anestésicocirúrgico, prestando assistência ao paciente juntamente com a equipe multiprofissional, ou seja, com o cirurgião, o anestesista e os técnicos de enfermagem, decidindo assim o melhor posicionamento para o paciente, facilitando as atividades durante o ato anestésico-cirúrgico. Desta forma, o enfermeiro identifica as alterações anatômicas e fisiológicas do paciente associadas ao tipo de anestesia, tempo cirúrgico e procedimento a que será submetido, para que não apresente complicações no pós-operatório 3 .

Os pacientes passam um longo tempo sobre a mesa cirúrgica, submetidos aos efeitos de analgésicos e relaxantes musculares, que embora sejam necessários, trazem ao paciente uma condição de fragilidade e dependência física ${ }^{4}$. Ainda existe a necessidade de colocá-lo e mantê-lo em variadas posições que atendam às exigências da técnica operatória e exponham o sítio cirúrgico; com isso, ações planejadas com atenção devem ser realizadas pela equipe de enfermagem, para que se obtenha sucesso no procedimento anestésico-cirúrgico ${ }^{1,3,5}$.

O posicionamento cirúrgico do paciente é um ato que exige competência e deve ser preciso e julgado como fator preponderante na realização segura do procedimento cirúrgico ${ }^{5}$, fator chave para a promoção do bem-estar e da segurança, prevenindo os eventos adversos ${ }^{6}$. Nesse contexto, para que o posicionamento seja realizado com qualidade, é primordial o planejamento da assistência, o trabalho em equipe e a utilização de dispositivos e equipamentos de posicionamento específicos para cada paciente ${ }^{3,4}$. Desta forma, são fundamentais os registros e a documentação de toda assistência prestada, mobilização, recursos de proteção utilizados, locais e condições clínicas do paciente ${ }^{2}$.

Cabe em especial ao enfermeiro implementar os cuidados que melhor atendam o paciente e saber reconhecer os fatores de risco relacionados ao posicionamento cirúrgico, para que se possam adotar medidas eficazes que contribuam na recuperação ${ }^{4}$.

$\mathrm{O}$ interesse por conduzir este estudo, ocorreu devido às observações em centro cirúrgico como instrumentadora cirúrgica verificando que as/os circulantes, algumas vezes, deixam de participar desse procedimento, quiçá por falta de embasamento científico sobre o posicionamento e as complicações que dele podem ocasionar se não for realizado de forma adequada. Isso alimentou a vontade de investigar na literatura, a fim de oferecer mais subsídios para a equipe de enfermagem das salas de operação.

Diante do exposto, o objetivo deste estudo é identificar na literatura os cuidados de enfermagem relacionados ao posicionamento cirúrgico do paciente adulto no transoperatório.

\section{MÉTODO}

O método de pesquisa adotado foi a revisão integrativa da literatura, um recurso da prática baseada em evidências, que possibilita a síntese e a análise do conhecimento científico já produzido sobre o tema investigado ${ }^{8}$. É um método que preconiza a utilização de resultados de pesquisas para a tomada de decisão do enfermeiro na sua prática diária ${ }^{8}$. Para a elaboração da revisão integrativa foram percorridas seis etapas, sobre as quais discorreremos a seguir.

A primeira etapa consistiu na formulação da pergunta norteadora: Quais os cuidados de enfermagem no posicionamento do paciente adulto no transoperatório?

Na segunda etapa foram realizadas pesquisas nos bancos de dados LILACS (Literatura Latino-Americana e do Caribe em Ciências da Saúde) e SciELO (Scientific Electronic Library Online), no período de 18 de agosto de 2004 a 30 de setembro de 2014, utilizando os descritores: "posicionamento do paciente" e "enfermagem". Os critérios de inclusão foram artigos publicados em língua portuguesa, com texto disponível na íntegra e a partir de 2004 e os critérios de exclusão artigos que não eram compatíveis com o objetivo desta revisão integrativa e os duplicados.

$\mathrm{Na}$ terceira etapa, ocorreu a extração das informações dos artigos para serem incluídos na revisão integrativa e a elaboração de um banco de dados com as informações pertinentes.

Nas quarta e quinta etapas, avaliaram-se os artigos e discutiram-se os tópicos que emergiram da leitura.

Finalmente, a sexta etapa contemplou a análise dos artigos selecionados. Ressalta-se que tanto a análise quanto a síntese das informações extraídas dos artigos foram feitas de forma descritiva, o que viabilizou observar, descrever e classificar as informações, com o propósito de reunir o conhecimento publicado sobre o tema eleito para esta revisão?. 


\section{RESULTADOS}

Com o descritor "posicionamento do paciente" foram encontrados 161 artigos, 112 na base de dados LILACS e 46 na base de dados SciELO. Combinando com o descritor "enfermagem", foram encontrados 20 artigos, 15 na base de dados LILACS e 2 na base de dados SciELO. Ainda foram encontrados três artigos publicados em revista específica sobre enfermagem em centro cirúrgico que foram utilizados nesta revisão, conforme demonstrados no Quadro 1. Dos 20 estudos encontrados foram utilizados 10 para a revisão integrativa, após aplicação dos critérios preestabelecidos, conforme demonstra o Quadro 2.

Dos $10(100 \%)$ estudos incluídos na revisão, constatou-se que $5(50 \%)$ se tratavam de pesquisa de campo com abordagem quantitativa, 3 (30\%) revisão da literatura, do tipo integrativa e $2(20 \%)$ revisão da literatura, do tipo sistemática. Em relação aos tipos de periódicos nos quais foram publicados, destacam-se os de enfermagem com o maior número de publicações, 9 (90\%). Quanto aos autores dos estudos, ficou evidenciado que todos são enfermeiros (100\% da amostra). Entre as localidades de origem das publicações, houve predomínio do estado de São Paulo, com 8 $(80 \%)$ artigos. O ano de 2011 foi o de mais publicação, com $4(40 \%)$ estudos.

Quadro 1. Resultado das buscas nas bases de dados por descritor - São Paulo, 2014.

\begin{tabular}{|l|c|c|c|c|}
\hline \multirow{2}{*}{ Descritores } & \multicolumn{4}{|c}{ Base de dados } \\
\cline { 2 - 6 } & LILACS & SciELO & Outras & Total \\
\hline $\begin{array}{l}\text { Posicionamento do paciente } \\
\begin{array}{l}\text { Posicionamento do paciente } \\
\text { e enfermagem }\end{array}\end{array}$ & 112 & 46 & 3 & 161 \\
\hline
\end{tabular}

Quadro 2. Apresentação da síntese dos artigos selecionados - São Paulo, 2014.

\begin{tabular}{|c|c|c|c|}
\hline $\begin{array}{l}\text { Autores } \\
\text { (ano) }\end{array}$ & $\begin{array}{l}\text { Periódico/ } \\
\text { localidade }\end{array}$ & $\begin{array}{l}\text { Tipo de } \\
\text { estudo }\end{array}$ & Síntese do artigo \\
\hline $\begin{array}{l}\text { Matos FGO, } \\
\text { Picoli M } \\
(2004)\end{array}$ & $\begin{array}{l}\text { Ciência, } \\
\text { Cuidado } \\
\text { e Saúde/ } \\
\text { Maringá (PR) }\end{array}$ & Quantitativo & $\begin{array}{l}\text { A amostra constituiu-se de } 30 \text { pacientes cirúrgicos de ambos os sexos, com idades entre } \\
20 \text { e } 60 \text { anos. Durante o acompanhamento dos pacientes no período transoperatório } \\
\text { foi identificado o diagnóstico de enfermagem Risco para Lesão Perioperatória por } \\
\text { Posicionamento em } 100 \% \text { da amostra. As principais recomendações são: cada sala } \\
\text { cirúrgica deve possuir um controle individual de temperatura para adequá-la às } \\
\text { necessidades do paciente, manter o paciente coberto para evitar desnecessária perda de } \\
\text { calor e fazer uso de soluções aquecidas. }\end{array}$ \\
\hline $\begin{array}{l}\text { Ursi ES, } \\
\text { Galvão CM } \\
\text { (2006) }\end{array}$ & $\begin{array}{l}\text { Rev. } \\
\text { Latino-Am. } \\
\text { Enfermagem/ } \\
\text { Ribeirão } \\
\text { Preto (SP) }\end{array}$ & $\begin{array}{l}\text { Revisão da } \\
\text { literatura } \\
\text { do tipo } \\
\text { integrativa }\end{array}$ & $\begin{array}{l}\text { A amostra constituiu-se de } 14 \text { artigos selecionados nas bases de dados CINAHL } \\
\text { e MEDLINE. Na revisão os resultados dos artigos apontaram que os dispositivos } \\
\text { considerados mais eficazes na prevenção de lesões de pele foram o colchão de ar } \\
\text { micropulsante, a cobertura de colchão de polímero de visco elástico seco e as } \\
\text { almofadas de gel. }\end{array}$ \\
\hline $\begin{array}{l}\text { Lopes CMM, } \\
\text { Galvão CM } \\
(2010)\end{array}$ & $\begin{array}{l}\text { Rev. } \\
\text { Latino-Am. } \\
\text { Enfermagem/ } \\
\text { Ribeirão } \\
\text { Preto (SP) }\end{array}$ & $\begin{array}{l}\text { Revisão da } \\
\text { literatura } \\
\text { do tipo } \\
\text { integrativa }\end{array}$ & $\begin{array}{l}\text { A amostra constituiu-se de } 20 \text { artigos selecionados nas bases de dados: PubMed, CINAHL e } \\
\text { LILACS. Todos os artigos avaliados apontaram que o posicionamento cirúrgico do paciente } \\
\text { causa algum impacto negativo nos sistemas do corpo e podem ocasionar complicações } \\
\text { como: dor musculoesquelética, deslocamento de articulações, danos em nervos } \\
\text { periféricos, lesões de pele, comprometimento cardiovascular e pulmonar e até síndrome } \\
\text { compartimental. }\end{array}$ \\
\hline $\begin{array}{l}\text { Scarlatti KC, } \\
\text { Michel LM, } \\
\text { Gambas MA, } \\
\text { Gutierrez } \\
\text { MGR (2011) }\end{array}$ & $\begin{array}{l}\text { Rev. Esc. } \\
\text { Enferm. } \\
\text { USP/São } \\
\text { Paulo (SP) }\end{array}$ & Quantitativo & $\begin{array}{l}\text { A amostra constituiu-se de } 199 \text { pacientes. Destes } 199,20,6 \% \text { apresentaram úlceras por } \\
\text { pressão, } 98,6 \% \text { nos estágios I e II, com localização predominante no tronco frontal }(35,1 \%) \text {. } \\
\text { As variáveis, posição, tempo cirúrgico, anestesia geral e uso de dispositivos apresentaram } \\
\text { associação estatística significativa. }\end{array}$ \\
\hline $\begin{array}{l}\text { Grigoleto } \\
\text { ARL, Avelar } \\
\text { MCQ, } \\
\text { Lacerda RA, } \\
\text { Mendonça } \\
\text { SHF (2011) }\end{array}$ & $\begin{array}{l}\text { Esc. Anna } \\
\text { Nery/São } \\
\text { Paulo (SP) }\end{array}$ & $\begin{array}{l}\text { Revisão da } \\
\text { literatura } \\
\text { do tipo } \\
\text { sistemática }\end{array}$ & $\begin{array}{l}\text { A amostra constituiu-se de sete estudos selecionados nas bases de dados PubMed/ } \\
\text { MEDLINE, Ovid, CINAHL, Cochrane e LILACS. Pode-se inferir que a responsabilidade do } \\
\text { enfermeiro com os demais membros da equipe, em sala de operações, está em avaliar } \\
\text { previamente o cliente como um todo, bem como observar as condições dos suportes } \\
\text { de apoio, o tempo de uso durante o procedimento e qualquer alteração que possa estar } \\
\text { relacionada ao posicionamento na mesa de operações. }\end{array}$ \\
\hline
\end{tabular}


Quadro 2. Continuação.

\begin{tabular}{|c|c|c|c|}
\hline $\begin{array}{l}\text { Autores } \\
\text { (ano) }\end{array}$ & $\begin{array}{l}\text { Periódico/ } \\
\text { localidade }\end{array}$ & $\begin{array}{l}\text { Tipo de } \\
\text { estudo }\end{array}$ & Síntese do artigo \\
\hline $\begin{array}{l}\text { Barbosa MH, } \\
\text { Oliva AMB, } \\
\text { Sousa Neto } \\
\text { AL (2011) }\end{array}$ & $\begin{array}{l}\text { Revista } \\
\text { Cubana de } \\
\text { Enfermería/ } \\
\text { Uberlândia } \\
\text { (MG) }\end{array}$ & Quantitativo & $\begin{array}{l}\text { A amostra constituiu-se de } 50 \text { pacientes adultos submetidos às cirurgias nas diferentes } \\
\text { especialidades. Observou-se que } 37(74,00 \%) \text { pacientes apresentaram lesões de pele } \\
\text { ao término do procedimento cirúrgico, sendo todas classificadas em Grau I. Compete à } \\
\text { enfermagem perioperatória a assistência ao paciente cirúrgico, sendo o posicionamento um } \\
\text { dos fatores fundamentais para a obtenção de um procedimento seguro e efetivo. }\end{array}$ \\
\hline $\begin{array}{l}\text { Carneiro GA, } \\
\text { Leite RCBO } \\
\text { (2011) }\end{array}$ & $\begin{array}{l}\text { Rev. Esc. } \\
\text { Enferm. } \\
\text { USP/São } \\
\text { Paulo (SP) }\end{array}$ & Quantitativo & $\begin{array}{l}\text { A amostra constituiu-se de } 182 \text { pacientes. A incidência de pacientes submetidos } \\
\text { à cirurgia cardíaca, que desenvolveram lesões de pele em decorrência do período } \\
\text { intraoperatório, foi de } 20,9 \% \text {. Tivemos que } 19,2 \% \text { das lesões apresentaram-se como } \\
\text { Úlceras por Pressão (UPP) no estágio I; } 1,1 \% \text { das lesões caracterizaram-se como } \\
\text { abrasão; } 1,1 \% \text {, feridas incisas; } 0,5 \% \text {, laceração; } 0,5 \% \text {, queimadura elétrica superficial e } \\
0,5 \% \text {, UPP no estágio II. Esse trabalho revela a incidência de lesões de pele no período } \\
\text { intraoperatório de uma pequena parcela de pacientes, mas incita os enfermeiros a } \\
\text { pensar com mais cuidado no tipo de assistência que ele vai oferecer ao paciente. }\end{array}$ \\
\hline $\begin{array}{l}\text { Rodrigues } \\
\text { RTF, Lacerda } \\
\text { RA, Leite } \\
\text { RB, Graziano } \\
\text { KU, Padilha } \\
\text { KG (2012) }\end{array}$ & $\begin{array}{l}\text { Rev. Esc. } \\
\text { Enferm. } \\
\text { USP/São } \\
\text { Paulo (SP) }\end{array}$ & $\begin{array}{l}\text { Revisão da } \\
\text { literatura } \\
\text { do tipo } \\
\text { integrativa }\end{array}$ & $\begin{array}{l}\text { A amostra constituiu-se de } 12 \text { artigos selecionados em bases de dados eletrônicas. A } \\
\text { análise dos artigos possibilitou concluir a pertinência de realização de estudos para avaliar, } \\
\text { principalmente, o impacto e a resolutividade dos recursos no atendimento às necessidades, } \\
\text { assim como melhoria ou geração de outros recursos inovadores. }\end{array}$ \\
\hline $\begin{array}{l}\text { Bentlin AC, } \\
\text { Grigoleto } \\
\text { ARL, Avelar } \\
\text { MCQ, } \\
\text { Sundfeld } \\
\text { MCK (2012) }\end{array}$ & $\begin{array}{l}\text { Rev. } \\
\text { SOBECC/São } \\
\text { Paulo (SP) }\end{array}$ & Quantitativo & $\begin{array}{l}\text { A amostra constituiu-se de } 14 \text { indivíduos idosos de ambos os sexos. Destes, } 6 \\
\text { apresentaram alterações na pele, as quais não estavam presentes na primeira etapa do } \\
\text { estudo (hiperemias, escoriações e hematomas). Esses idosos permaneceram em mesa } \\
\text { operatória entre } 50 \text { e } 130 \text { minutos, sem os devidos recursos de proteção. }\end{array}$ \\
\hline $\begin{array}{l}\text { Sergio FR, } \\
\text { Cameron } \\
\text { LE, Vital ICO } \\
(2012)\end{array}$ & $\begin{array}{l}\text { Rev. } \\
\text { SOBECC/São } \\
\text { Paulo (SP) }\end{array}$ & $\begin{array}{l}\text { Revisão da } \\
\text { literatura } \\
\text { do tipo } \\
\text { sistemática }\end{array}$ & $\begin{array}{l}0 \text { artigo traz a importância da atuação do enfermeiro no reconhecimento precoce das } \\
\text { complicações decorrentes do posicionamento cirúrgico, contribuindo na prevenção } \\
\text { de agravos. Apresenta como fatores de risco para o desenvolvimento da síndrome } \\
\text { compartimental: obesidade, doença vascular periférica, cirurgias prolongadas (maior que } \\
3 \text { horas) e a posição litotômica. }\end{array}$ \\
\hline
\end{tabular}

\section{DISCUSSÃO}

Durante a avaliação dos artigos, constatou-se que três tópicos principais foram focados, a saber:

1. fatores de risco para o desenvolvimento de complicações;

2. complicações decorrentes do posicionamento cirúrgico;

3. cuidados de enfermagem relacionados ao posicionamento cirúrgico do paciente.

A presente revisão integrativa fornece aos profissionais da saúde informações detalhadas sobre os tópicos mencionados.

\section{Fatores de risco para 0 desenvolvimento de complicações}

As condições preexistentes devem ser consideradas ao planejar a assistência de enfermagem ao paciente cirúrgico, especialmente as que afetam os sistemas vascular, respiratório, circulatório, neurológico e imunitário 5 . Todos os fatores de risco devem ser identificados na avaliação pré-operatória e documentados, contribuindo para o plano de cuidados ${ }^{3}$.

Os pacientes cirúrgicos são os primeiros candidatos a desenvolverem lesões de pele no intraoperatório, devido à diminuição de fluxo sanguíneo capilar, por tempo prolongado de imobilidade e pressão ${ }^{2}$. Uma das complicações mais comuns é o desenvolvimento de Úlcera por Pressão (UPP).

Existem diversos fatores de risco relacionados à etiopatogenia da UPP que se desenvolvem durante os procedimentos cirúrgicos e podem ser agrupados em fatores intrínsecos e extrínsecos. Dentre os intrínsecos, destacam-se: idade (pessoas muito jovens e idosos podem ter a pele mais sensível), peso corporal (o sobrepeso e o baixo peso podem potencializar o surgimento de lesões), estado nutricional (desnutrição e desidratação) e doenças crônicas, como diabetes mellitus, vasculopatias, neuropatias, hipertensão e anemia. 
Dentre os extrínsecos, tipo e tempo de cirurgia (procedimentos maiores que duas horas podem comprometer a oxigenação dos tecidos comprimidos), anestesia (perda da proteção fisiológica compensatória), problemas no controle da temperatura corporal (hipotermia faz com que estruturas do corpo dependam de mais oxigênio e, sem o aporte necessário, pode favorecer o aparecimento de lesões), posições cirúrgicas e imobilização devido ao posicionamento.

Algumas posições associadas a particularidades do paciente podem aumentar o risco de complicações, como a posição de litotomia em um paciente obeso, que poderá comprometer sua função respiratória, podendo causar até síndrome compartimental. Sendo assim, todos os pacientes cirúrgicos devem ser considerados como de alto risco para o desenvolvimento de $\mathrm{UPP}^{3,7,10}$.

\section{Complicações decorrentes do posicionamento cirúrgico}

O volume do fluxo de sangue capilar pulmonar diminui com a imobilidade prolongada. A expansão pulmonar é limitada pela pressão da posição sobre as costelas ou pela capacidade de o diafragma forçar o conteúdo abdominal para baixo. A anestesia provoca vasodilatação periférica, resultando em hipotensão e diminuindo o retorno venoso. Faz ainda com que as defesas normais diminuam a capacidade de proteção contra a manipulação excessiva ${ }^{2}$.

Sem dúvida, a pele enquanto barreira natural é o órgão que está mais sujeito aos agravos decorrentes do posicionamento cirúrgico, apresentando eritemas, equimoses, risco para UPP, queimaduras elétricas, lesões por substâncias químicas e alopecia focal ${ }^{4}$. O tecido e a pele podem ficar lesionados devido ao excesso de pressão ou hematomas decorrentes de contato com superfície dura. A pressão externa de $32 \mathrm{mmHg}$ é considerada o limiar; além desta, os pequenos vasos entram em colapso, causando trombose, o que resulta em oclusão do fluxo sanguíneo tecidual e privação da quantidade necessária de oxigênio e nutrientes. Ocorre, então, a produção de metabólitos tóxicos a nível celular, levando a acidose tecidual, aumento da permeabilidade capilar, edema, morte celular e formação de UPP ${ }^{3}$. As UPPs, como qualquer lesão causada por pressão não aliviada que resulta em danos aos tecidos, ocorrem geralmente nas regiões de proeminências ósseas e são graduadas em estágios I, II, III e IV. Além das UPPs, outros eventos agressivos estão presentes no ambiente cirúrgico passíveis de atingir o paciente, como as queimaduras elétricas devido ao uso do eletrocautério8.
Os estudos analisados demonstraram que o posicionamento cirúrgico do paciente pode causar algum impacto negativo nos sistemas do corpo, ocasionando várias complicações, como: dor músculo-esquelética, deslocamento de articulações, danos em nervos periféricos, lesões de pele, comprometimento cardiovascular e pulmonar e até síndrome compartimental ${ }^{3}$.

A Síndrome Compartimental apesar de estar mais frequentemente associada ao trauma tem sido relatada como consequência do posicionamento cirúrgico durante cirurgias prolongadas. Caracteriza-se por um aumento na pressão dentro de um compartimento corporal, reduzindo a perfusão capilar até abaixo do nível necessário para a viabilidade dos tecidos, levando à isquemia, podendo ocasionar um déficit neuromuscular permanente se houver demora no diagnóstico e no tratamento, que consiste na realização de fasciotomia descompressiva ${ }^{7}$.

A presença ou a instalação de processos patológicos podem resultar em alterações que provocam o comprometimento da sua estrutura funcional e, consequentemente, aumento da estadia hospitalar para seu tratamento ${ }^{5}$.

\section{Cuidados de enfermagem relacionados ao posicionamento cirúrgico do paciente}

O posicionamento do paciente para a cirurgia é essencial para o desempenho de um procedimento seguro e eficiente, por isso, todos os membros da equipe devem protegê-lo de qualquer efeito danoso decorrente do inadequado posicionamento. Compete ao enfermeiro, juntamente com os demais membros da equipe, a responsabilidade de avaliar previamente o paciente como um todo, observar as condições dos suportes de apoio e qualquer situação que possa comprometer o posicionamento do paciente na mesa de operações e acarretar complicações ${ }^{5,7}$.

As intervenções eficazes na prevenção de lesões de pele estão relacionadas ao alívio de pressões durante e imediatamente após a permanência do paciente na mesa cirúrgica, sobre o colchão padrão ${ }^{5}$. Os dispositivos mais eficazes na prevenção de lesões de pele, em ordem decrescente, são: o colchão de ar micro pulsante, a cobertura de colchão de polímero de visco elástico seco e as almofadas de gel, sucessivamente ${ }^{8}$.

O uso dos recursos de proteção no posicionamento do paciente no intraoperatório assegura a manutenção da integridade da pele e das pressões osteoarticulares e neuromusculares, tendo como objetivo evitar atritos, prevenindo lesões da pele como UPPs, compressão ou estiramentos neuromusculares, contato com o metal da mesa que pode ocasionar queimaduras (devido ao uso do bisturi elétrico) e outros danos ${ }^{6}$. 
Os estudos analisados trazem os cuidados de enfermagem no posicionamento cirúrgico do paciente e recomendações de acordo as posições na mesa cirúrgica, listados a seguir.

Decúbito dorsal ou supina: acolchoar o calcâneo, o sacro, o cóccix, o olecrano, a escápula, a tuberosidade isquiática e o occipital. Manter os braços nas laterais com as palmas voltadas para baixo (em pronação) ou "em cruz" e o braço apoiado à tala, em um ângulo inferior a $80^{\circ}$ em relação ao corpo, para não provocar estiramento dos músculos e nervos nessa região, assim como a compressão das artérias subclávia e axilar sob o processo coroide da escápula ou a compressão entre a clavícula e as primeiras duas costelas. Não garrotear as extremidades para fixar as mãos. O lençol da mesa cirúrgica pode ser utilizado para fixar os braços em toda a sua extensão e prevenir que fiquem, se for o caso, pendentes ao longo da mesa. A cabeça deve estar alinhada à coluna vertebral e ao quadril. Um pequeno coxim abaixo da cabeça permite o relaxamento do músculo trapézio e previne a distensão do pescoço; flexões e torções podem causar contraturas e interferir na permeabilidade das vias aéreas. Os membros inferiores (MMII) devem permanecer estendidos e os pés, ligeiramente separados ${ }^{3,6}$.

Decúbito ventral ou prona: proteger o quadril e as coxas com travesseiros grandes. Proteger os seios. Acomodar a genitália masculina em posição lateral. Proteger o dorso dos pés, a cintura escapular, o olecrano, a espinha ilíaca e a patela ${ }^{3}$.

SIMS (lateral): manter o alinhamento espinhal, observar orelhas, colocar um apoio sob a cabeça, a região da axila e entre as pernas, manter a perna em contato com a mesa flexionada na região do quadril e a superior esticada. Usar uma fita adesiva de $10 \mathrm{~cm}$ de largura fixada a um lado da mesa, sobre a crista ilíaca, até o outro lado ${ }^{2,3}$.

Litotomia: utilizar acolchoamento nos estribos. Ter duas pessoas para levantar lentamente as pernas com leve rotação do quadril, posicionar os joelhos com leve flexão. Cobrir as pernas com botas de algodão. Posicionar os braços sobre as talas ou relaxados sobre o abdome, apoiados por um lençol. Reverter o posicionamento lentamente, para favorecer a adaptação fisiológica do organismo à nova posição, uma vez que a mudança brusca pode causar hipotensão severa devido à súbita alteração do fluxo circulatório. Revestir as perneiras metálicas com coxins macios, com altura ajustada ao comprimento dos MMII do paciente, posicionando os dois membros ao mesmo tempo, ou seja, dois profissionais executando o procedimento, atentando-se ainda em realizá-lo de formas segura e lenta, flexionando os joelhos e apoiando uma das mãos na região da planta dos pés ${ }^{3,6}$.
Cada sala cirúrgica deve possuir um controle individual de temperatura para adequá-la às necessidades do paciente, devendo manter a temperatura corporal acima de $35,5^{\circ} \mathrm{C}^{1}$.

Vale ressaltar que há limitações neste estudo devido à ausência de artigos específicos sobre o tema e atuais. Considera-se importante a realização de novos estudos utilizando-se dos dispositivos de proteção para comprovar a sua eficácia na prevenção de complicações decorrentes do posicionamento do paciente na mesa de cirurgia.

\section{CONCLUSÃO}

Conclui-se que é de vital importância os cuidados de enfermagem no transoperatório, tendo visto que a avaliação do paciente é realizada considerando os fatores de risco para o desenvolvimento de complicações que possam comprometer a sua integridade física. Diversos fatores podem contribuir para o surgimento de eventos adversos, entre eles alguns se referem às particularidades do paciente, como idade, peso, condição nutricional e doenças pré-existentes. O enfermeiro, como profissional responsável pelo paciente no centro cirúrgico, deve garantir a sua proteção e segurança, valendo-se da SAEP (Sistematização da Assistência de Enfermagem Perioperatória), ferramenta disponível, indispensável e de alta importância, que permite uma melhor avaliação, bem como, uma assistência totalitária de acordo com as necessidades do paciente, composta por fases distintas, mas que, se bem desenvolvidas dispõem de uma assistência de enfermagem individualizada e de qualidade; essas fases são: anamnese e exame físico, diagnóstico de enfermagem, plano assistencial, prescrição de enfermagem e, consequentemente, a evolução de enfermagem baseada em uma assistência correta.

É importante que o enfermeiro esteja preparado para prevenir as complicações que possam ocorrer, desde queimaduras e lesões de pele até o comprometimento de nervos e tecidos devido ao inadequado posicionamento na mesa cirúrgica ou mesmo a uma hipotensão severa ocasionada pela resposta fisiológica à posição, pois uma vez que ocorram e não sejam diagnosticadas rapidamente, poderão causar sérios danos ao paciente.

Cabe ao enfermeiro promover ações para a prevenção de complicações decorrentes do procedimento anestésicocirúrgico, acompanhar o paciente em todas as etapas do seu tratamento, inclusive disponibilizar todos os recursos necessários para o sucesso do procedimento, supervisionando todas as ações da equipe de enfermagem. Para isso, faz-se necessário que se atualize constantemente e exerça a educação continuada com seus colaboradores. 


\section{REFERÊNCIAS}

1. Matos FGO, Piccoli M. Diagnóstico de Enfermagem Risco para Lesão Perioperatória por Posicionamento identificado no período transoperatório. Rev Ciência, Cuidado e Saúde 2004;3(2):195-201.

2. Carneiro GA, Leite RCBO. Lesões de pele no intraoperatório de cirurgia cardíaca: incidência e caracterização. Rev Esc Enferm USP 2011; 45(3):611-6. [acesso em 18 ago 2014]. Disponível em: http:// www.scielo.br/pdf/reeusp/v45n3/v45n3a09.pdf

3. Lopes $\mathrm{CMM}$, Galvão $\mathrm{CM}$. Posicionamento cirúrgico: evidências para o cuidado de enfermagem. Rev Latino-Am Enferm. 2010;18(2):[08 telas]. [acesso em 18 ago 2014]. Disponível em: http://www.scielo. $\mathrm{br} / \mathrm{pdf} / \mathrm{rlae} / \mathrm{v} 18 \mathrm{n} 2 / \mathrm{pt} \_21 . p d f$

4. Barbosa MH, Oliva AMB, Sousa Neto AL. Ocorrência de lesões perioperatórias por posicionamento cirúrgico. Revista Cubana de Enfermería 2011;27(1):31-41.

5. Grigoleto ARL, Avelar MCQ, Lacerda RA, Mendonça SHF. Complicações decorrentes do posicionamento cirúrgico de clientes idosos submetidos à cirurgia de quadril. Esc Anna Nery (impr.) 2011;15(3):531-5.

6. Bentlin AC, Grigoleto ARL, Avelar MCQ. Lesões de pele decorrente do posicionamento cirúrgico no cliente idoso. RevSOBECC. 2012;17(2):56-63.
7. Sérgio FR, Cameron LE, Vital ICO. Síndrome Compartimental relacionada ao posicionamento cirúrgico: um inimigo silencioso. Rev SOBECC. 2012;17(3):71-80.

8. Ursi ES, Galvão CM. Prevenção de lesões de pele no perioperatório: Revisão integrativa da literatura. Rev Latino-Am Enferm. 2006;14(1):124-31. [acesso em 18 ago 2014]. Disponível em: http:// www.scielo.br/pdf/rlae/v14n1/v14n1a17.pdf

9. Polit DF, Beck CT, Hungler BP. Fundamentos da pesquisa em Enfermagem: métodos, avaliação e utilização. 5 ed. Porto Alegre (RS); 2004.

10. Scarlatti KC, Michel LM, Gambas MA, Gutierrez MGR. Úlcera por pressão em pacientes submetidos à cirurgia: incidência e fatores associados. Rev Esc Enferm USP. 2011;45(6):1372-9. [acesso em 18 ago 2014]. Disponível em: http://www.scielo.br/pdf/reeusp/v45n6/ v45n6a14.pdf

11. Rodrigues RTF, Lacerda RA, Leite RB, Graziano KU, Padilha, KG. Enfermagem transoperatória nas cirurgias de redução de peso: revisão integrativa da literatura. Rev Esc Enferm USP. 2012;46(Esp):138-47. [acesso em 18 ago 2014]. Disponível em: http://www.scielo.br/pdf/ reeusp/v46nspe/20.pdf 